\title{
Zinc Mediated Barbier Type Allylation of Cyclic Imides and Subsequent Coupling Reactions with Carbon Nucleophiles
}

\author{
Seok Won Kang, Eun Young Heo, Jong Gab Jun,; and Sung Hoon Kim ${ }^{\star}$ \\ Department of Chemistry, Konkuk Chivensity Seoul 143-701. Korea \\ ${ }^{\dagger}$ Department of Chemistrv, Hallw Unversin, Chunchon 200-702, Korea \\ Received September IS, 2004
}

\begin{abstract}
Allylations of $N$-benzyl and $N$-methyl cyclic imides were accomplished successfully under mild Barbier type conditions using zinc metal, allyl bromide and catalytic amount of $\mathrm{PbBr}_{2}$. Subsequent coupling reactions with some carbon nucleophiles afforded 1.2- and 1,4-addition products in moderate to ligh yields.
\end{abstract}

Key Words : Ally lation, Cyclic imide. Barbier. $N$-Acyliminiun ion. Carbon nucleopliles

\section{Introduction}

Recently. Barbier type ally lation reactions are extensively studied with aldehyde. ${ }^{1}$ ketone. ${ }^{2}$ imine ${ }^{3}$ acetal ${ }^{4}$ and nitrile. but Barbier type allylation with cyclic imides have not been well studied in the literature ${ }^{6}$ to our knowledge. Reported allylation reactions of cyclic imides have been restricted within 5-membered ring and organomagnesium. organolithium ${ }^{6}$ or organozine reagent ${ }^{7}$ under anhy'drous condition with moderate y ields. As part of our interest in the chemistry of cyclic $N$-acyliminium ions. we reported that the zinc mediated Barbier type propargylation of cyclic imides proceeded well. ${ }^{\S}$ Recently, we found that zinc mediated Barbier type allylation of cyclic imides also proceeded very well to give allylated hydroxy lactames without the formation of allylidene compounds or recovery of starting materials. Furthermore. we found that $i n$ situ generated $\mathrm{N}$ acyliminium ions can be reacted with carbon nucleophiles such as allyl tri-h-butyltin. trimethylsilyl cyanide and 2.4pentanedione to introduce second substituents to the lactams (Scheme 1).

\section{Results and Discussion}

First. we wish to summarize the results of Barbier type allylation of cyclic imides. To a mixture of cyclic imides. metals. catalyst and solvent was added allyl bromide. Among the metals such as In. $\mathrm{Zn} . \mathrm{Mg}$. $\mathrm{Sn} . \mathrm{Fe} . \mathrm{Cu} . \mathrm{Pb}, \mathrm{Al}$ and catalysts such as $\mathrm{ZnCl}_{2} . \mathrm{MgBr}_{2} . \mathrm{TiCl}_{4} . \mathrm{SnCl}_{4} . \mathrm{PbBr}_{2}$. $\mathrm{GaCl}_{\hat{3}}$ and $\mathrm{BF}_{3} \cdot \mathrm{Et}_{2} \mathrm{O}$. combination of $\mathrm{Zn}$ and $\mathrm{PbBr}_{\hat{\imath}}$ gave best

Table 1. Zinc mediated Barbier type allylation of cyclic imides<smiles>[Y]N1C(=O)CCC1=O</smiles><smiles>C=CCBr</smiles>

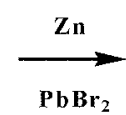

2<smiles>[Z1]N1C(=O)CCC1=CC=C</smiles><smiles>[R1]NC(=O)CCC(C)(O)CC=C</smiles>

5

$\mathbf{R}_{1}=\mathrm{CH}_{3}$, benzyl, $\quad \mathrm{n}=0,1,2$

\begin{tabular}{|c|c|c|c|c|c|c|}
\hline \multirow{2}{*}{ entry } & \multirow{2}{*}{$\begin{array}{l}\text { cyclic } \\
\text { imides }\end{array}$} & \multirow{2}{*}{$\mathrm{R}_{\mathrm{l}}$} & \multirow{2}{*}{ solvent } & \multicolumn{3}{|c|}{ isolated yield } \\
\hline & & & & 3 & 4 & 5 \\
\hline 1 & $n=1$ & Bn & THF: $\mathrm{Et}_{2} \mathrm{O}=1: 1, \pi \mathrm{t}$ & 78 & 10 & 3 \\
\hline 2 & $n=1$ & Bn & THF, rt & 50 & - & 28 \\
\hline 3 & $n=1$ & Bn & THF, $0-5^{\circ} \mathrm{C}$ & 88 & - & - \\
\hline 4 & $\mathrm{n}=\mathrm{l}$ & $\mathrm{CH}_{3}$ & THF, rt & 60 & 30 & - \\
\hline 5 & $n=1$ & $\mathrm{CH}_{3}$ & 1,4-dioxane, rt & 65 & 21 & - \\
\hline 6 & $n=1$ & $\mathrm{CH}_{3}$ & $\mathrm{THF},-5-0^{\circ} \mathrm{C}$ & 86 & - & - \\
\hline 7 & $n=2$ & $\mathrm{Bn} 1$ & THE, rt & - & - & 85 \\
\hline 8 & $\mathrm{n}=2$ & $\operatorname{Bn} 1$ & $\mathrm{THF}=5-0^{\circ} \mathrm{C}$ & - & - & 98 \\
\hline 9 & $n=3$ & Bn & THF, it & - & - & 90 \\
\hline 10 & phthalimide & Bin & THF $0.5^{\circ} \mathrm{C}$ & 95 & - & - \\
\hline
\end{tabular}

All reactions were carried out $2.5-5 \mathrm{mmol}$ scale, 2 equiv of zinc granule and 2-2.5 equiv, of allyl bromide were used.

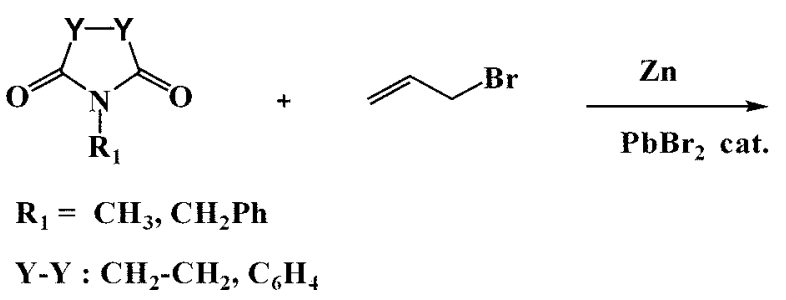<smiles>[R1]n1c(C)[Y](C=C):[Y]([Y])(O)c1=O</smiles>

Scheme 1 
results. Actually, the use of $\mathrm{PbBr}_{\beth}$ as cataly'st was essential to ensure the completion of the reaction to provided ally lated $\alpha$-hy'droxy lactams in high yields without the recovery of cyclic imides. It was reported that the use of chemically activated zinc increased the yields of Reformatsky reaction ${ }^{1 \text { tab }}$ and pulsed sono-electrochemically produced zinc powder greatly increased yield of Barbier type allylation. ${ }^{1, x}$ However. zinc granule purchased from Aldrich without further activation gave good results in our experiments. The hydroxy-lactams $3^{11}$ were exclusively obtained when the reactions were carried out at $0-5{ }^{\circ} \mathrm{C}$. Higher temperature. strong Lewis acid catalyst or quenching with $0.5 \mathrm{M} \mathrm{HCl}$ led to increased formation of ally lidenes 4 . Also. hydroxy-lactam 3 can be easily converted to 4 in the presence of acid catalyst. Aftempts to purify the product by silica gel chromatography led to slight decomposition to allylidene 4 . In the case of larger cyclic imides such as $\mathrm{N}$ - benzylglutarimide and $\mathrm{N}$-benzyladipinide. major products are ring-opened di-allylated compounds $\mathbf{5}^{12}$ These results are sunmmarized in Table 1.

All reactions were carried out $2.5-5 \mathrm{mmol}$ scale. 2 equiv. of zinc granule and 2-2.5 equiv. of allyl bromide were used.

Second. we wish to report the results of alkylation of Barbier type allylation product with carbon nucleophiles. Reactions between $N$-acyliminium ions and nucleophiles have been frequently utilized to introduce substituents at the $\alpha$-carbon of an amine. ${ }^{12}$ The addition of Grignard reagents and organolithium species to cyclic imide. followed by reaction with $\mathrm{Et}_{3} \mathrm{SiH}_{1}{ }^{14}$ allyltributy $1 \mathrm{ltin}^{\text {ic }}$ has been studied. However. to the best of our knowledge. efforts regarding the creation of a $\alpha \alpha$-diallylation and cyanation through the addition of a second carbon nucleophile are not studied well. So. we have examined in situ alky lation reactions of 3-allyl2-benzyl-3-hydroxy-2.3-dihydroisoindol-1-one (6) with

Table 2. Coupling reactions of ally lated $k$-benzylphthalimide

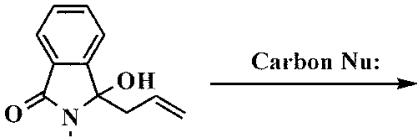

$\mathrm{Bn}$

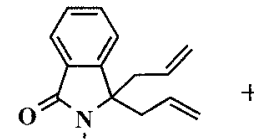

$\mathrm{Bn}$<smiles>CCCC1(C)NC(=O)c2ccccc21</smiles>

Bn

6

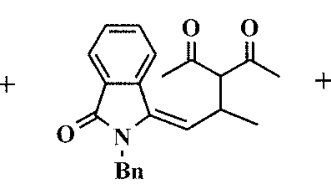

9

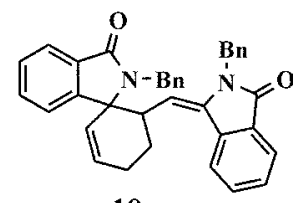

10

\begin{tabular}{|c|c|c|c|}
\hline Entry & carbon nucleophile & reaction condition & isolated yield \\
\hline 11 & allyltributyltin & $\mathrm{BF}_{3} \mathrm{OEt}_{2}, \mathrm{MC},-78^{\circ} \mathrm{C} \rightarrow 0^{\circ} \mathrm{C}, \mathrm{ld}$ & $7(98 \%)$ \\
\hline 12 & trimethylsilyl cyanide & $\mathrm{BF}_{3} \mathrm{OEt}_{2}, \mathrm{MC}, 0^{\circ} \mathrm{C} \rightarrow \mathrm{rt}, 1.5 \mathrm{~h}$ & $8(45 \%)$ \\
\hline $13 \mathrm{a}$ & 2,4-pentanedione & $\mathrm{BF}_{3} \mathrm{OEt}_{2}, \mathrm{MC},-78^{\circ} \mathrm{C} \rightarrow 0^{\circ} \mathrm{C}, \mathrm{ld}$ & $9(18 \%)$ \\
\hline $13 b$ & 2,4-pentanedione & $120^{\circ} \mathrm{C}$, neat condition, $2 \mathrm{~h}$ & $9(33 \%)+10(65 \%)$ \\
\hline $13 \mathrm{c}$ & - & $120^{\circ} \mathrm{C}, \mathrm{AcOH}, \mathrm{THF}, 2 \mathrm{~h}$ & $10(93 \%)$ \\
\hline
\end{tabular}<smiles>O=C1c2ccccc2C(=O)N1Cc1ccccc1</smiles><smiles></smiles>

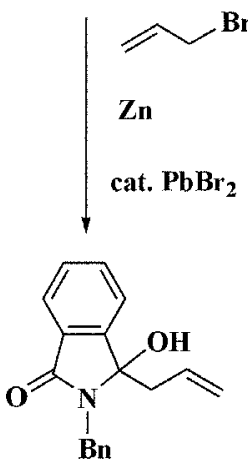

6
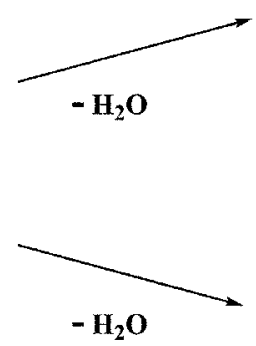

Carbon Nucleophile

1,t-addition

2,4-pentanedione

1<smiles>C=CCC1=C(Cc2ccccc2)N(Cc2ccccc2)C1=O</smiles><smiles>C=C/C=C1\c2ccccc2C(=O)N1Cc1ccccc1</smiles><smiles>CC(N)/C=C1\c2ccccc2C(=O)N1Cc1ccccc1</smiles>

Carbon Nucleophile

1,2-addition

TMS-CN, Tributylallyltin

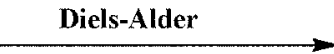<smiles>O=C1C2=C(CCC=C2)N(Cc2ccccc2)/C1=C\CC1(C2=CC=CCC2)C=CCc2ccccc21</smiles>

Scheme 2 
Table 3. Coupling reaction with ally lated N-benzylsuccinimide<smiles>C=CCBr</smiles>

3<smiles>C=CC=C1CCC(=O)N1Cc1ccccc1</smiles>

4

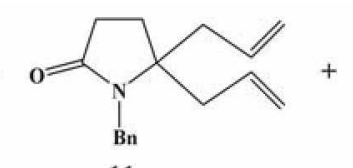

11

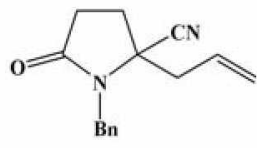

12<smiles>CC(=O)C1C(=O)C2CC(=O)N(O)C2=CC1C</smiles>

13

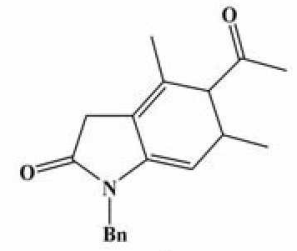

14

\begin{tabular}{|c|c|c|c|}
\hline Entry & Carbon Nucleophile & reaction condition & isolated yield \\
\hline 14 & allỵltributỵltin & $\mathrm{BF}_{2} \cdot \mathrm{OEt}_{2}, \mathrm{MC}_{3}-78^{\circ} \mathrm{C} \rightarrow 0^{\circ} \mathrm{C}, \mathrm{Id}$ & $4(51 \%)+11(23 \%)$ \\
\hline 15 & trimethylsilyl cranide & $\mathrm{BF}_{3}, \mathrm{OEt}_{2}, \mathrm{MC}, 0^{\circ} \mathrm{C} \rightarrow \mathrm{rt}, 1.5 \mathrm{~h}$ & $12(55 \%)$ \\
\hline $16 a$ & 2,4-pentanedione & $55^{\circ} \mathrm{C}, \mathrm{THF}, 3 \mathrm{~d}$ & $13(58 \%)$ \\
\hline $16 \mathrm{~b}$ & 2,4-pentanedione & $120^{\circ} \mathrm{C}$, neat condition, $1 \mathrm{~d}$. & $13(29 \%)+14(32 \%)$ \\
\hline $16 \mathrm{c}$ & 2,4-pentanedione & $55^{\circ} \mathrm{C}, \mathrm{AcOH}, \mathrm{THF}, \mathrm{I} \mathrm{d}$ & $13(32 \%)+14(64 \%)$ \\
\hline
\end{tabular}

All reaction was preceded by addition for concened, in sinn generated $\gamma$ hydroxy lactam 3 .

carbon nucleophiles such as allyltributyltin. ${ }^{\text {(N) }}$ trimethylsilyl cyanide ${ }^{13}$ and 2.4-pentanedione to give 1.2- and 1.4-addition products (Table 2)

The $\mathrm{BF}_{3} \mathrm{OEt}_{2}$ promoted the addition of allyltributyltin. trimethylsilyl cyanide to 6 afforded 5.5-disubstituted isoindolones. On the other hand other results were observed from the addition of $\beta$-diketone. Under similar condition. slow addition reaction proceeded via the formation of immediate $N$-acyliminium ion. rearranged to more stable conjugated $N$-acyliminium ion to give 1.4 -addition product 9 in $18 \%$ yield (entry 13a). Reaction of 6 in 2.4-pentanedione at $120^{\circ} \mathrm{C}$ gave $9^{15}$ and dimeric product 10 (entry 13b). Upon heating of 6 to $120^{\circ} \mathrm{C}$ in acetic acid dimeric compound $10^{16}$ was obtained in good yield. The stnucture of $\mathbf{1 0}$ is prove to be Diels-Alder product (entry 13c. 1: 1 mixture of endoand exo-products). (Scheme 2).

In another set of experiments. in situ alkylation reactions of allylated $\mathrm{N}$-benzylsuccinimide 3 were tested with nucleophiles such as allyltributyltin. trimethylsilyl cyanide and 2.4-pentanedione to give 1.2- and 1.4-addition products (Table 3). Enamide formation 4 arise. if the $N$-acyliminium ion is not trapped fast enough by a nucleophile.

All reaction was preceded by addition for concemed in situ generated $\gamma$ hỵdroxy lactam 3.
On the other hands. another results were observed in the addition of $\beta$-diketone with enol content to in sim generated fhydroxy lactam 3 at $55^{\circ} \mathrm{C}$ giving alkylation product $13^{17}$ in $58 \%$ yield (entry 16a). Upon heating to $120^{\circ} \mathrm{C}$. a mixture of two adducts $13: 14^{18}$ was obtained in $61 \%$ yield. Also. in the presence of acetic acid a mixture of two adducts $13: 14$ was increased quantitatively in $96 \%$ y ield (entry 16c).

To confirm the structure of cyclization product 14 , the reaction mixture was treated with 2 eq of DDQ to give 5acetyl-1-benzyl-3-hydroxy-4.6-dimethyl-1.3-dihy droindol2-one 15 with $76 \%$ yield ${ }^{19}$ (Scheme 3 ).

\section{Experimental Section}

Allylation of cyclic imides. To a mixture of $\mathrm{N}$-benzylsuccinimide (5 mmol), zinc granule $(10 \mathrm{mmol})$ and catalytic amount of $\mathrm{PbBr}_{2}$ in THF $(10 \mathrm{~mL})$ was added ally bronide (10 mmol) slowly at $0-5^{\circ} \mathrm{C}$. stirred for $5 \mathrm{~h}$ until it became sticky greenish gray slurry. The reaction mixture was quenched with aq. $\mathrm{NH}_{4} \mathrm{Cl}$ solution $(30 \mathrm{~mL})$, then extracted with methylene chloride $(2 \times 50 \mathrm{~mL})$. The methylene chloride solution was washed with brine $(10 \mathrm{~mL})$. dried over sodiun sulfate. filtered and concentrated to give almost pure $3(88 \%)$ which was crystallized out from ether/hexane to<smiles>C=CC(=O)C1C(=O)CC2CC(=O)N([Hg])C2=CC1C</smiles>

13<smiles>CC(=O)C(CC1=CCC(=O)N1)C(C(C)=O)C(C)=O</smiles>

$-\mathrm{H}_{2} \mathrm{O}$<smiles>CC(=O)C1CC=C2C(=C(C)CC(=O)N2[12CH2])C1</smiles>

$\underset{\mathrm{DDQ}}{\stackrel{2 \text { eq. }}{\longrightarrow}}$

14<smiles>CC(=O)c1ccc2c(c1C)C(O)C(=O)N2[12CH3]</smiles>

15

Scheme 3 
give as a white solide (entry 3): mp $88^{\circ} \mathrm{C}:{ }^{1} \mathrm{H}$ NMR $(400$ $\left.\mathrm{MHz}, \mathrm{CDCl}_{3}\right) \delta$ 7.1-7.3 (m. 5H). 5.4-5.5 (m. 2H). 4.9-5.0 (m. $2 \mathrm{H}), 4.6(\mathrm{~d} .1 \mathrm{H}), 4.3(\mathrm{~d}, \mathrm{lH}), 2.4(\mathrm{~m}, 2 \mathrm{H}), 1.9-2.2(\mathrm{~m}$. $4 \mathrm{H}) .{ }^{13} \mathrm{C}$ NMR $\left(100 \mathrm{MHz}, \mathrm{CDCl}_{3}\right) \delta 176.2 .138 .7 .132 .4$. 128.8. 128.4 127.5. 119.7.92.4. 44.4. 42.8. 32.2.29.5.

To a mixture of $N$-benzylglutarimide ( $1 \mathrm{mmol}$ ), zinc granule $(2.5 \mathrm{mmol})$ and catalytic amount of $\mathrm{PbBr} \_$in THF $(10 \mathrm{~mL})$ was added ally bromide $(2.5 \mathrm{~mm}$ ) slowly at $0-5$ ${ }^{\circ} \mathrm{C}$, stirred for $5 \mathrm{~h}$ until it became sticky greenish gray slurry. The reaction mixture was quenched with aq. $\mathrm{NH}_{4} \mathrm{Cl}$ solution $(10 \mathrm{~mL})$, then extracted with methylene chloride $(2 \times 20$ $\mathrm{mL}$ ). The methylene chloride solution was washed with brine $(10 \mathrm{~mL})$. dried over sodium sulfate. filtered and concentrated to give almost pure 5 ( $98 \%$. entry 8$)$ : ${ }^{l} \mathrm{H}$ NMR $\left(400 \mathrm{MHz}, \mathrm{CDCl}_{3}\right): \delta 1.45-1.49(\mathrm{~m}, 2 \mathrm{H}), 1.83(\mathrm{~m}, 2 \mathrm{H}), 2.20-$ $2.25(\mathrm{~m} .6 \mathrm{H}) .4 .4(\mathrm{~d} .2 \mathrm{H}) .5 .08-5.13(\mathrm{~m} .4 \mathrm{H}) .5 .79-5.86(\mathrm{~m}$. $2 \mathrm{H}) .7 .26-7.34(\mathrm{~m} .5 \mathrm{H}) ;{ }^{13} \mathrm{C}$ NMR $\left(100 \mathrm{MHz}, \mathrm{CDCl}_{3}\right) \delta$ 19.8, 37.0. 38.6, 43.9, 44.0, 119.1, 127.8, 128.2. 129.0. 134.0 .138 .7 .173 .1 .

To a mixture of $\mathrm{N}$-benzy'ladipimide ( $1 \mathrm{mmol}$ ), zinc granule (2.5 mmol) in THF $(10 \mathrm{~mL}$ ) was added ally bromide $(2.5$ mmol) slowly at $0-5{ }^{\circ} \mathrm{C}$. stirred for $5 \mathrm{~h}$ until it became sticky greenish gray slurry. The reaction mixture was quenched with aq. $\mathrm{NH}_{4} \mathrm{Cl}$ solution $(10 \mathrm{~mL})$. then extracted with methylene chloride $(2 \times 20 \mathrm{~mL})$. The methylene chloride solution was washed with brine $(10 \mathrm{~mL})$. dried over sodium sulfate. filtered and concentrated to give almost pure $\mathbf{5}(90 \%$. entry 9): ${ }^{l} \mathrm{H}$ NMR $\left(400 \mathrm{MHz}, \mathrm{CDCl}_{3}\right) \delta 1.34-1.43(\mathrm{~m} .4 \mathrm{H})$. $1.58-1.62$ (q. $2 \mathrm{H}$ ). $2.16-2.20$ (m. $6 \mathrm{H}$ ). 4.3 (d. $2 \mathrm{H}) .5 .05-5.11$ (m. $4 \mathrm{H}), 5.75-5.84(\mathrm{~m}, 2 \mathrm{H}), 6.45$ (s. $1 \mathrm{H}), 7.22-7.30(\mathrm{~m}, 5 \mathrm{H})$ : ${ }^{13} \mathrm{C} \mathrm{NMR}\left(100 \mathrm{MHz}, \mathrm{CDCl}_{3}\right) \delta 22.9 .26 .1,36.4 .38 .6,43.4$. $43.6 .73 .3,118.5,127.3 .127 .7,128.6,133.8,138.5,173.1$.

Coupling reactions of allylated $\mathrm{N}$-benzylphthalimide. To a solution of $6(5 \mathrm{mmol})$ in methylene chloride $(25 \mathrm{~mL})$. under an argon atmosphere at $-78^{\circ} \mathrm{C}$ was added allyl tri-nbutyltin followed by $\mathrm{BF}_{3} \mathrm{OEt}_{2}$ dropwise. The reaction mixture was quenched with $5 \% \mathrm{NaHCO}_{3}$ solution $(30 \mathrm{~mL})$. then extracted with methylene chloride $(2 \times 50 \mathrm{~mL})$. The methylene chloride layer was washed with brine $(10 \mathrm{~mL})$. dried over sodium sulfate. filtered and concentrated to give almost pure diallylated product 7 ( $98 \%$. entry 11 ): $\mathrm{mp} 92-93$ ${ }^{\circ} \mathrm{C}:{ }^{1} \mathrm{H}$ NMR $\left(400 \mathrm{MHz}, \mathrm{CDCl}_{3}\right) \delta 7.4(\mathrm{~m}, 4 \mathrm{H}) .7 .2(\mathrm{~m} .5 \mathrm{H})$. $4.9(\mathrm{~m}, 2 \mathrm{H}), 4.6(\mathrm{~d}, 2 \mathrm{H}), 4.5(\mathrm{~m}, 4 \mathrm{H}), 2.5(2.4 \mathrm{H}):{ }^{13} \mathrm{C} \mathrm{NMR}$ $\left(100 \mathrm{MHz}, \mathrm{CDCl}_{3}\right) \delta 169.5,147.8 .138 .7 .132 .5,132.0$. 131.4, 129.4. 129.1, 128.8, 128.5, 127.8. 122.1, 121.1. $119.5 .69 .4,43.4 .41 .9$.

To a solution of $6(2.01 \mathrm{mmol})$ in methylene chloride( 10 $\mathrm{mL}$ ). under an argon atmosphere at $-3{ }^{\circ} \mathrm{C}$ was added trimethylsilyl cyanide followed by $\mathrm{BF}_{3} \mathrm{OEt}_{2}$ dropwise. The reaction mixture was quenched with sat. $\mathrm{Na}_{2} \mathrm{CO}_{3}$ solution $(10 \mathrm{~mL})$, then extracted with methylene chloride $(2 \times 20$ $\mathrm{mL}$ ). The methylene chloride layer was washed with brine $(10 \mathrm{~mL})$. dried over sodium sulfate. filtered and concentrated to give $\alpha \alpha$-disubstituted product $8(45 \%$. entry 12$)$ : ${ }^{l} \mathrm{H}$ NMR (400 MHz. $\left.\mathrm{CDCl}_{3}\right) \delta 7.8($ d. $1 \mathrm{H}) .7 .5(\mathrm{~m} .3 \mathrm{H}) .7 .3(\mathrm{~d}$. $2 \mathrm{H}) .7 .2(\mathrm{~m} .3 \mathrm{H}) .5 .0(\mathrm{~m} .1 \mathrm{H}) .4 .9$ (d. $2 \mathrm{H}) .4 .7(\mathrm{~m} .2 \mathrm{H}) .2 .8-$ 2.6 (m. 2H): ${ }^{13} \mathrm{C} \mathrm{NMR}\left(100 \mathrm{MHz}, \mathrm{CDCl}_{3}\right) \delta 168.0 .141 .7$.
$136.5,133.3,130.9,130.7,129.1,128.5,127.8,124.8$. $122.8,122.7,117.2,61.8,45.1,41.7$.

Coupling reaction with allylated $N$-benzylsuccinimide. To a solution of 3 in methylene clloride. under argon atmosphere at $-78^{\circ} \mathrm{C}$ was added allyltributyltin followed by $\mathrm{BF}_{3} \mathrm{OEt}_{2}$ dropwise. The reaction mixture was quenched with $5 \%$. $\mathrm{NaHCO}_{3}$ solution $(30 \mathrm{~mL})$, then extracted with metlylene chloride $(2 \times 50 \mathrm{~mL})$. The methylene chloride solution was washed with brine $(10 \mathrm{~mL})$, dried over sodium sulfate. filtered and concentrated to give 5-allylidene-1benzylpyrrolidin-2-one 4 in $51 \%$ yield and the formation of addition product 11 in $23 \%$ yield (entry 14 ). ${ }^{1} \mathrm{H}$ NMR (400 $\left.\mathrm{MHz}, \mathrm{CDCl}_{3}\right) \delta 7.27-7.13(\mathrm{~m} .5 \mathrm{H}), 5.4(\mathrm{~m}, 2 \mathrm{H}) .4 .95$ (dd. $4 \mathrm{H}) .4 .3(\mathrm{~d} . \mathrm{lH}), 4.4$ (s. $2 \mathrm{H}), 2.3(\mathrm{~m} .2 \mathrm{H}) .2 .2(\mathrm{~m} .2 \mathrm{H}), 2.0$ (m. 2H), $1.8(\mathrm{~m}, 2 \mathrm{H}) \cdot{ }^{13} \mathrm{C}$ NMR (100 MHz, $\left.\mathrm{CDCl}_{2}\right) \delta 176.2$. $139.0,132.7,128.8 .128 .6 .127 .6,119.7 .72 .2,66.4,43.9$. 43.6. 30.2. 27.8

To a solution of 3 ( $1 \mathrm{mmol}$ ) in methylene chloride (10 $\mathrm{mL}$ ). under argon atmosphere at $-78{ }^{\circ} \mathrm{C}$ was added trimethylsilyl cyanide $(3 \mathrm{mmol})$ followed by $\mathrm{BF}_{3} \mathrm{OEt}_{2}$ dropwise. The reaction mixture was quenched with $5 \%$. $\mathrm{NaHCO}_{3}$ solution $(30 \mathrm{~mL})$, then extracted with methylene chloride $(2 \times 50 \mathrm{~mL})$. The methylene chloride solution was washed with brine $(10 \mathrm{~mL})$. dried over sodium sulfate. filtered and concentrated to give $\alpha \alpha$-disubstituted product 12 in $55 \%$ yield (entry 15 ): ${ }^{1} \mathrm{H}$ NMR ( $\left.400 \mathrm{MHz}, \mathrm{CDCl}_{2}\right) \delta$ $7.3(\mathrm{~m} .5 \mathrm{H}) .5 .5(\mathrm{~m} . \mathrm{lH}) .5 .2(\mathrm{dd} .2 \mathrm{H}) .4 .8(\mathrm{~d} .1 \mathrm{H}), 4.4(\mathrm{~d}$ lH). $2.6(\mathrm{~m}, 3 \mathrm{H}), 2.4(\mathrm{~m}, \mathrm{lH}), 2.26(\mathrm{~m}, \mathrm{lH}), 2.25(\mathrm{~m} .1 \mathrm{H})$ : ${ }^{13} \mathrm{C}$ NMR $\left(100 \mathrm{MHz}, \mathrm{CDCl}_{3}\right): \delta 174.9,136.8,129.1,128.6$, $128.4,122.6 .119 .5,72.2 .61 .6,45.3 .42 .8,30.4 .29 .1$.

\section{Conclusions}

We have demonstrated a efficient and convenient method of Barbier type allylations of cyclic imides was accomplished under mild condition in excellent yields and applied to the further in sim reactions with various carbon nucleophiles to afford in highly fnctionalized 5.5-disubstituted isoindolones. spirocyclization compound. We also found that $\beta$-diketone as carbon nucleophile can be used as precursors for a $\pi$-nucleophilic attack onto allylated hydroxy lactanss. giving 1.2-addition products. So. allylated hydroxy lactams have proven a useful $N$-acyliminium ion precursor in coupling reaction. We are currently studying reactions of allylated hydroxy lactams with some other carbon nucleophiles in order to investigate their utilization in the total șynthesis of natural compounds.

Acknowledgements. This work was financially supported by Konkuk university 2001.

\section{References}

1. (a) Zhou. T.-Y.: Tia. Y.: Sutr. G.F.: Wu. S.-H. Sym. Conmmm 1997. 27. 1899. (b) Basu. M. K.: Banik. B. K. Tetrahedron Lett. 2001. 42. 187. (c) Cleghom. L. A. T.: Cooper. I. R.: Grigg. R.: MacLachlan. W. S.; Sridharan, V. Tetrahedon Lett 2003. H4. 7969. (d) Cha, J. H.; Cho. Y. S.; Koh. H. Y: Lee, E.: Kim. Y. T: 
Yang. H. H.: Kang. H. Y. Bull. Konean Chem. Soc. 2004. 25(8). 1123. (e) Cho. Y. S.: Kang. K. H.: Cha. J. H.: Choi. K. I.: Pae. A. N.: Koh. H. Y.: Chang. M. H. Bull. Korean Chent. Soc. 2002. 23(9). 1285.

2. (a) Auge, I: Lubin-Germain, N.: Thiaw-Woave, A. Tetrahedron Lett. 1999, t0. 9245. (b) Lee, P. H.: Seomoon. D.: Lee. K. Bull. Korem (them. Soc, 2001, 22(12). 1380.

3. (a) Wang. D. K.: Dai. L. X.: Hou. X. L: Zhang. Y. Tetrahedron Lett. 1996. 37. 4187, (b) Legros J. : Meyer F. J. Org. Chem. 2003. 68.6447

4. Tanaka. H.: Nakahata. S.: Watanabe, H.: Zhao. J.: Kuroboshi, M: Torii, S. Inorganica Chimica Acta 1999. 296.204

5. Lee, A. S.-Y.: Lin, L.-S. Tetrahetron Lett. $2000,41,8803$.

6. El-Nezhawy. A. O. H.: El-Diwani. H. I.: Schmidt. R. R. Eur. J. Org. Chem. 2002. 4137.

7. (a) Yoda. H: Yamazaki. H.: Takale. K. Tetrahedron Astmmem 1995, 6, 2669. (b) Yada. H.: Kitavama H: Yamada. W.: Takabe. K. Tethathedron Asymmeny 1993, A. 1451. (c) Schuch, C. M.: Pilli. R. A. Tetrahedron: Assmmeny 2000. 11.753. (d) Pearson. W. H: Dietz. A.: Stoy, P. Org. Lett. 2004. 6(6). 1005.

8. Kim. S. H.: Han. E. Y. Tetrahedron Lett. 2000 . H. 6479.

9. Tanaka. H.: Yamashita. S.: Ikemoto. Y: Torii. S. Chem. Lett. 1987, 673 .

10. (a) Picotin. G.: Miginiac. P. J. Ong. Chem 1987. 52, 4796. (b) Benincasa. M.: Forti, L.: Ghelfi, F. Symth. Commm. 1996, 26. 4113. (c) Durant. A.: Delplancke. J. L.: Winand. R.: Reisse. J. Tetrahedron Lett. 1995. 36.4257.

11. $\mathrm{mp} 88^{\circ} \mathrm{C}$ : NMR data of 5-allyl-1-benzyl-5-hydroxypyrrolidin-2one 3: ${ }^{1} \mathrm{H} \mathrm{NMR}\left(400 \mathrm{MHz} . \mathrm{CDCl}_{3}\right)$ o 7.1-7.3 (m. $\left.5 \mathrm{H}\right) .5 .4-5.5(\mathrm{~m}$. $2 \mathrm{H}) \cdot 4.9-5.0(\mathrm{~m} .2 \mathrm{H}) \cdot 4.6(\mathrm{~d}, 1 \mathrm{H}), 4.3(\mathrm{~d} .1 \mathrm{H}), 2.4(\mathrm{~m} .2 \mathrm{H}) .1 .9-2.2$ (m, 4H); ${ }^{1} \mathrm{C}$ NMR $\left(100 \mathrm{MHz}, \mathrm{CDCl}_{3}\right) \delta 176.2,138.7 .132 .4$. 128.8. 128.4. 127.5. 119.7, 92.4, 44.4, 42.8, 32.2.29.5.

12. (a) Cristina M. Schuch.: Ronaldo A. Pilli. Tetrahedron Astrmmetry 2002.13. 1973

13. Oba. M.: Koguchi. S.: Nishiyama. K. Tetrahedron 2002. 58.9359.

14. Yoda. H.: Shimojo, T: Takabe, K. Tetrahedron Lett 1999, 40, 1335.

15. NMR data of 3-[2-(2-benzy 1-3-oxo-2-3-dilydroisoindol-1-ylidene)1-methylethyl]pentane-2 4-dione (9) ${ }^{\mathrm{l}} \mathrm{H}$ NMR $(400 \mathrm{MHz}$.
$\left.\mathrm{CDCl}_{3}\right): \delta 7.9(\mathrm{dd} .2 \mathrm{H}), 7.5(\mathrm{td} .2 \mathrm{H}), 7.3-7.1(\mathrm{~m}, 5 \mathrm{H}) .5 .0(\mathrm{dd} .2 \mathrm{H})$ 4.9 (d. IH). 3.9 (m. IH). 3.5 (d. IH). 2.1 (s. $3 \mathrm{H}$ ). 1.7 (s. $3 \mathrm{H}$ ). 0.9 (d. $3 \mathrm{H}):{ }^{13} \mathrm{C}$ NMR (100 MHz. CDCl $\left.l_{3}\right) \delta 203.2$. 166.8. 137.2. 135.4. 135.2. 132.8. 130.9. 129.7. 129.1. 127.8. 127.2. 124.2. $114.2,75.9 .43 .3 .32 .7 .30 .6 .29 .7 .19 .9$.

16. NMR data of Diels-Alder product 10: (a) ${ }^{1} \mathrm{H}$ NMR $(400 \mathrm{MHz}$. $\left.\mathrm{CDCl}_{3}\right) 88.0(\mathrm{~d} .1 \mathrm{H}), 7.7(\mathrm{~d} . \mathrm{lH}) .7 .6(\mathrm{~m} .2 \mathrm{H}) .7 .3-7.1(\mathrm{~m} .6 \mathrm{H})$ 6.8 (m. $3 \mathrm{H}$ ). 6.7 (d. $2 \mathrm{H}$ ). 6.6 (t. $2 \mathrm{H}$ ). 6.4 (t. $1 \mathrm{H}$ ). 6.0 (m. $1 \mathrm{H}$ ). 5.0 (d. IH). 4.9 (d. $1 \mathrm{H}) .4 .5$ (dd. $2 \mathrm{H}$ ). 4.3 (d. $1 \mathrm{H}$ ). 4.0 (d. $1 \mathrm{H}$ ). 3.2 (m. $\mathrm{lH}) .2 .3$ (m. 2H). 2.1 (m. IH). 1.7 (m. $\mathrm{lH}$ ). ${ }^{13} \mathrm{C} \mathrm{NMR}(100 \mathrm{MHz}$. $\left.\mathrm{CDCl}_{3}\right) \& 170.5,166.4 .150 .4,139.2 .136 .6,135.9 .1350,133.1$ $132.6,132.3 .131 .0 .130 .9,129.3 .129 .1,128.9 .128 .8,128.6$. $127.8,127.7 .127 .6 .126 .9,124.3 .124 .2 .122 .9,121.6 .111 .3,67.8$ 60.8. 46.1. 43.1. 41.8. 26.2. 25.4. 21.5. 14.6. (b) ${ }^{1} \mathrm{H}$ NMR (400 $\mathrm{MHz} . \mathrm{CDCl}$ ) $\delta 8.1$ (d. $\mathrm{lH}) .7 .9$ (d. $1 \mathrm{H}) .7 .8(\mathrm{~m} .2 \mathrm{H}) .7 .6(\mathrm{t.} \mathrm{lH}$ ). 7.5-7.4 (m. 1IH). $7.2(\mathrm{~m} .2 \mathrm{H}) .6 .5(\mathrm{~m} . \mathrm{lH}) .5 .4$ (d. $1 \mathrm{H}) .5 .2$ (dd. $2 \mathrm{H}) .5 .1(\mathrm{~d}, \mathrm{lH}) .4 .7(\mathrm{~d}, \mathrm{lH}) .4 .6(\mathrm{~d}, \mathrm{lH}) .3 .9(\mathrm{~m}, \mathrm{lH}) .2 .6(\mathrm{~m} .2 \mathrm{H})$. $2.0(\mathrm{~m} .3 \mathrm{H})$.

17. NMR data of 3-[1-methyl-2-(5-oxo-1-phenylpyrolodin-2-ylidene)ethyl]pentane-2.4-dione (13): 'H NMR (400 MHz. CDCli): $\delta 7.1$ $7.2(\mathrm{~m} .5 \mathrm{H}) .5 .0$ (d. IH). 4.2 (d. IH). 4.1 (d. $1 \mathrm{H}$ ). 3.3 (d. IH). 2.9 (m. $\mathrm{lH}$ ). 2.6 (m. $2 \mathrm{H}$ ). 2.5 (m. $2 \mathrm{H}$ ). 2.0 (s. $3 \mathrm{H}$ ). 1.6 (s. $3 \mathrm{H}$ ). 0.8 (d. $3 \mathrm{H}){ }^{13} \mathrm{C}$ NMR $\left(100 \mathrm{MHz} . \mathrm{CDCl}_{3}\right): \delta 200.6,202.8 .175 .7 .140 .1$. $135.9,128.6 .128 .4,127.3 .126 .9,103.5 .75 .2,53.5 .43 .4,33.1$ $31.1,29.1,28.6,21.1,19.7$

18. NMR data of 5-acetyl-1-benzy-4.6-dimethyl-1.3.5.6-tetrahydroindol-2-one (14): ${ }^{1} \mathrm{H} \mathrm{NMR}$ (400 MHz. CDCl 3 ) $\delta 7.2-7.1$ (m. 5H). 4.7 (d. $1 \mathrm{H}) .4 .5$ (d. $1 \mathrm{H}) \cdot 4.4$ (d. $1 \mathrm{H}) .3 .1$ (s. $2 \mathrm{H}) \cdot 2.7$ (s. $1 \mathrm{H}$ ). 2.6 $(\mathrm{m} .1 \mathrm{H}), 2.0(\mathrm{~s}, 3 \mathrm{H}), 1.6(\mathrm{~s} .3 \mathrm{H}), 0.8(\mathrm{~d} .3 \mathrm{H}),{ }^{13} \mathrm{C} \mathrm{NMR}(100 \mathrm{MHz}$. $\left.\mathrm{CDCl}_{3}\right) \delta 200.3,173.5 .138 .2,136.2 .128 .9,127.6 .127 .5,125.4$. $122.8,97.6 .62 .0 .44 .0,33.2 .31 .5 .28 .6,22.4$. 18.9. Mass, $\mathrm{M}^{-}$ (295), M-15 (280), M-43 (252)

19. NMR data of 5-acetyl-1-benzyl-3-hydroxy-4.6-dimethyl-1.3-dihydroindol-2-one (15): 'H NMR ( $400 \mathrm{MHz}$. CDCl $)$ ): $\delta 7.3$ (m. $5 \mathrm{H}$ ). 6.4 (s. $1 \mathrm{H}$ ). 5.1 (s. $1 \mathrm{H}$ ). 4.9 (d. $\mathrm{lH}$ ). 4.8 (d. IH). 2.4 (s. $3 \mathrm{H}$ ). $2.3(\mathrm{~s} .3 \mathrm{H}), 2.1(\mathrm{~s}, 3 \mathrm{H}), 1.5(\mathrm{~s} .1 \mathrm{H}),{ }^{13} \mathrm{C}$ NMR $\left.(100 \mathrm{MHz}, \mathrm{CDCl})_{3}\right)$ $\delta 207.1 .172 .4,143.4,138.3,136.1,135.1,131.9 .129 .1,128.0$. $127.2,121.7,109.4,51.2,41.1,32.6 .20 .1 .15 .8$ 